Supporting Information

\title{
Quantitative evaluation of fullerene separation by
}

\section{liquid chromatography}

\author{
Sebastian Süß $\beta^{a, b}$, Vanessa Michaud ${ }^{a, b}$, Konstantin Amsharov ${ }^{c}$, Vladimir Akhmetov ${ }^{c}$, \\ Malte Kaspereit ${ }^{d}$, Cornelia Damm ${ }^{a, b}$ and Wolfgang Peukert ${ }^{a, b, *}$
}

${ }^{a}$ Institute of Particle Technology (LFG), Friedrich-Alexander-Universität Erlangen-Nürnberg (FAU), Cauerstr. 4, 91058 Erlangen, Germany.

${ }^{\mathrm{b}}$ Interdisciplinary Center for Functional Particle Systems (FPS), Friedrich-Alexander-Universität Erlangen-Nürnberg (FAU), Haberstraße 9a, 91058 Erlangen, Germany.

${ }^{c}$ Chair of Organic Chemistry II, Friedrich-Alexander-Universität Erlangen-Nürnberg (FAU), Nikolaus-Fiebiger-Str. 10, 91058 Erlangen, Germany.

${ }^{\mathrm{d}}$ Institute of Separation Science and Technology (TVT), Friedrich-Alexander-Universität Erlangen-Nürnberg (FAU), Egerlandstr. 3, 91058 Erlangen, Germany.

Corresponding Author:

* E-mail: Wolfgang.Peukert@fau.de; Phone: +49 91318529401. 


\section{CONTENT:}

S1. Peak areas and peak widths of fullerene separation ................................................. 3

S2. Chromatograms of fullerene separation for $\mathrm{He}$ coefficient evaluation ..................... 4

S3. Chromatograms of fullerene separation with varying flow rate............................. 5

S4. Retention time, peak width and resolution in dependence on flow rate for the

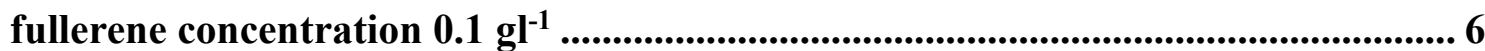




\section{S1. Peak areas and peak widths of fullerene separation}

a)

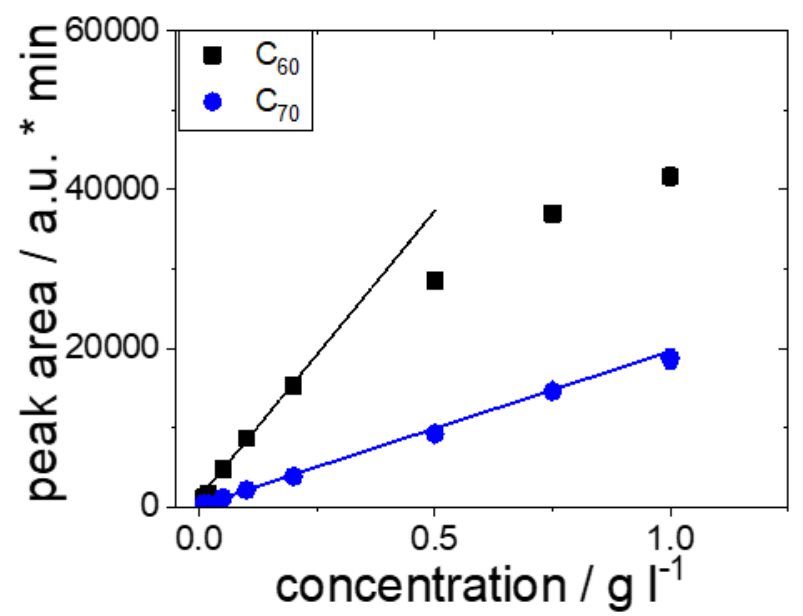

b)

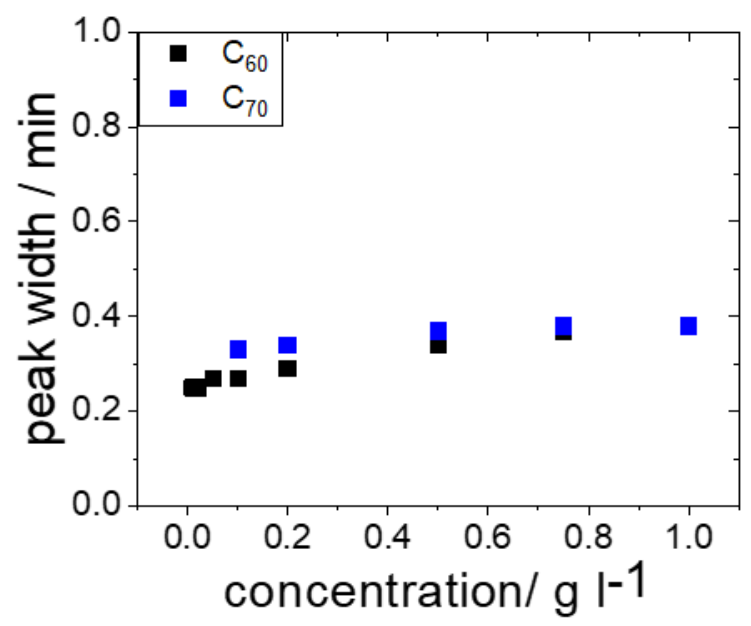

Figure S1: $\quad$ a) Peak areas b) Peak width for $C_{60}$ and $C_{70}$ depending on the total fullerene concentration $\left(0.01 \mathrm{gl}^{-1}-1 \mathrm{gl}^{-1}\right)$ for two injections of $20 \mu \mathrm{l}$ sample each at a flow rate of $1 \mathrm{ml} \mathrm{min}^{-1}$ using toluene as mobile phase and a detection wavelength of $300 \mathrm{~nm}$. 
S2. Chromatograms of fullerene separation for $\mathrm{He}$ coefficient evaluation

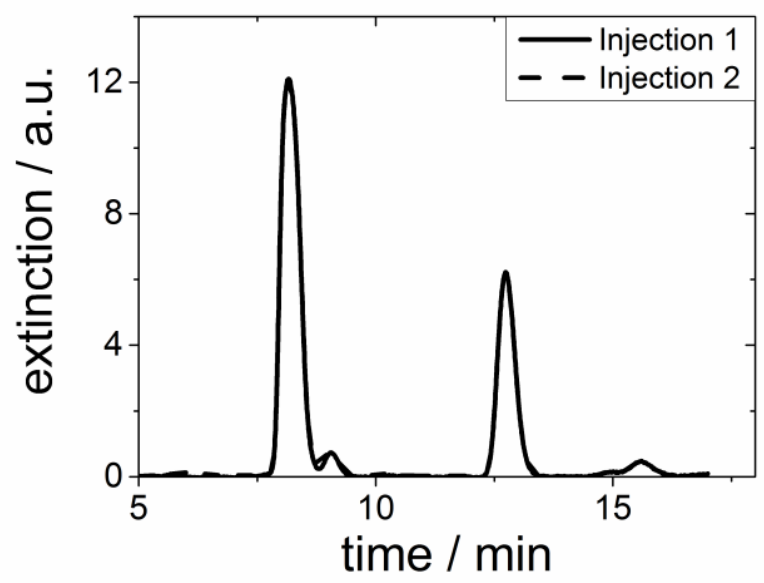

Figure S2: $\quad$ Chromatograms of $C_{60} / C_{70}$ fullerene mixture for two injections of each $20 \mu \mathrm{l}$ sample (concentration $1 \mathrm{~g}^{-1}$ ) at a flow rate of $1 \mathrm{ml} \mathrm{min}^{-1}$ using toluene as mobile phase and a detection wavelength of $300 \mathrm{~nm}$. 


\section{S3. Chromatograms of fullerene separation with varying flow rate}

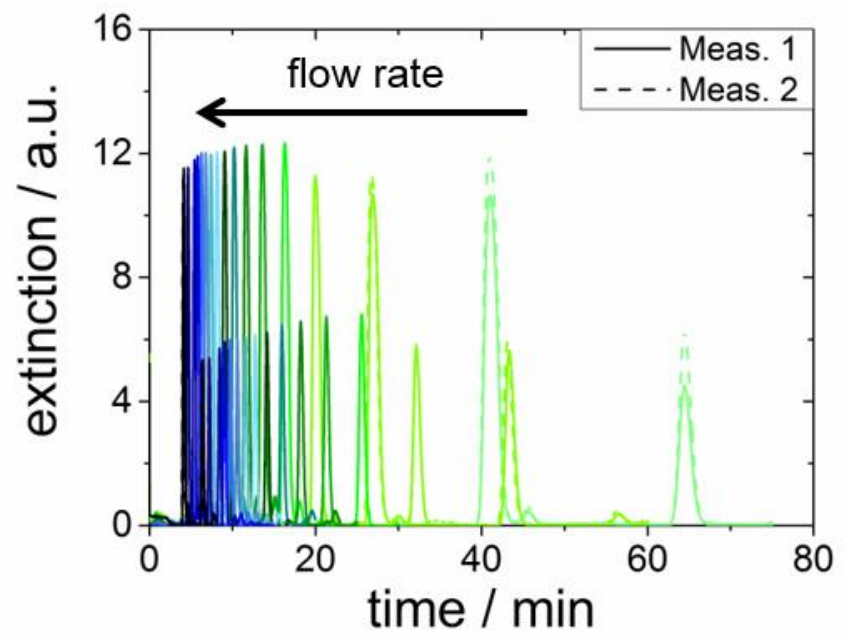

Figure S3: $\quad$ Chromatograms of fullerene mixture with varying flow rate $\left(0.2 \mathrm{ml} \mathrm{min}^{-1}-\right.$

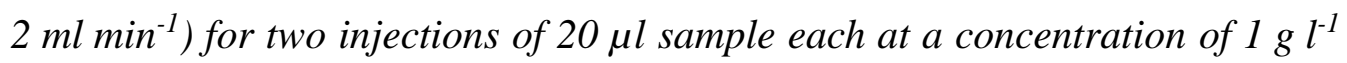
using toluene as mobile phase and a detection wavelength of $300 \mathrm{~nm}$. 
S4. Retention time, peak width and resolution in dependence on flow rate for the fullerene concentration $0.1 \mathrm{gl}^{-1}$

a)

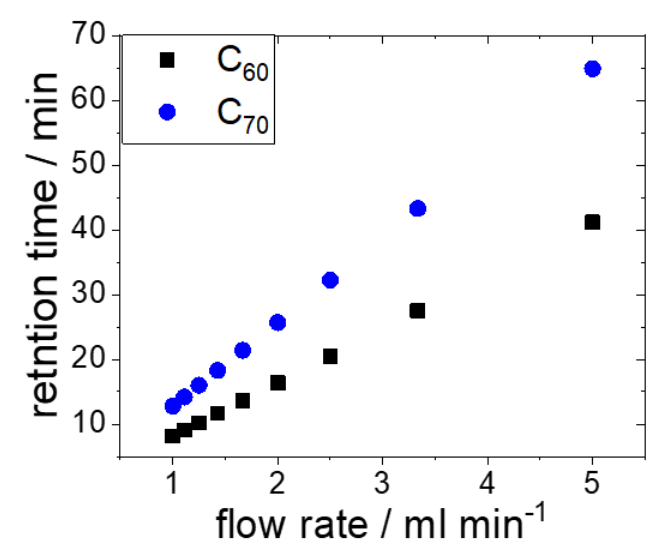

b)

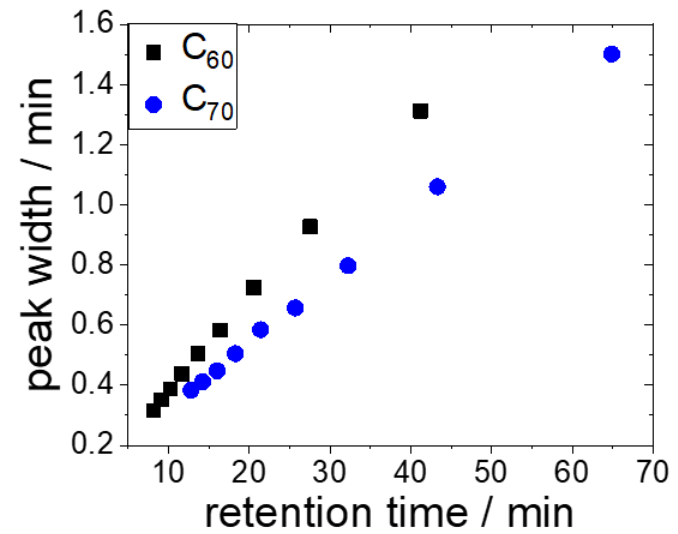

c)

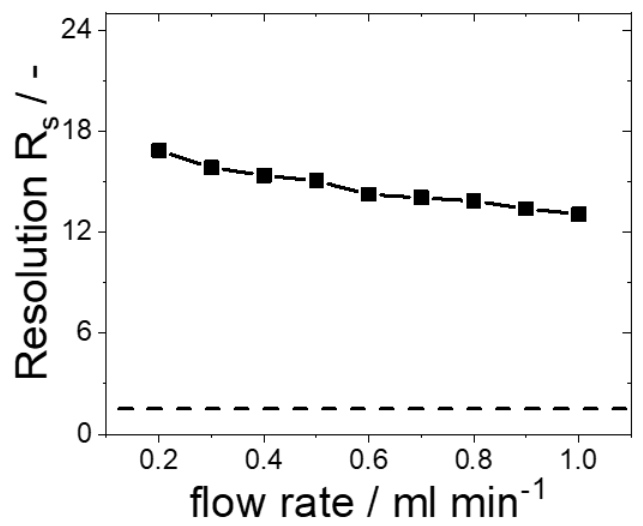

Figure S4: (a) Retention time and (b) peak width at the basis of the peak of $C_{60}$ (black squares) and $C_{70}$ (blue dots) fullerene mixture (concentration $0.1 \mathrm{~g}^{-1}$ ) depending on the flow rate for two injections of $20 \mu \mathrm{l}$ sample using toluene as mobile phase and a 
detection wavelength of $300 \mathrm{~nm}$. (c) Resolution $R_{s}$ of $C_{60}$ and $C_{70}$ separation depending on the flow rate. 